the central nervous system. To date, however, evidence of a unique immunologic abnormality in patients with multiple sclerosis is lacking. In particular, $T$ cells that recognize myelin can be identified with similar frequency in patients with and without multiple sclerosis.

Studies on the disease have suggested a noticeable heterogeneity in disease pathogenesis ${ }^{3}$; at least 4 different histologic patterns of the lesion were shown. For example, in some cases, the process seems to be directed primarily at the myelin sheath, with relative sparing of oligodendrocytes, whereas in other cases, there is primary destruction of oligodendrocytes. Although the lesion of multiple sclerosis is primarily inflammatory and demyelinating, recent evidence reemphasizes that axonal loss may occur early in the disease course and that it is critically important in the development of irreversible disability.

\section{MAGNETIC RESONANCE IMAGING}

Magnetic resonance imaging (MRI) has both improved the diagnostic accuracy of multiple sclerosis and played an important role in better understanding the natural history of the disease. Patients with frequent relapses often exhibit new lesions after enhancement with gadolinium, indicating focal breakdown of the blood-brain barrier. Secondary or primary progression is associated with markers of tissue destruction, as shown by MRI (increased volume of T1 hypointense lesions, reduced magnetization transfer ratio, progressive atrophy), rather than new activity of focal lesions.

In addition, magnetic resonance imaging has prognostic and therapeutic applications: the amount of lesions in the early phases of the disease predicts future disability, and quantification of disease activity (lesions shown after enhancement with gadolinium, new lesions) and lesion burden provides a powerful tool in therapeutic trials. ${ }^{4,5}$

\section{CLINICAL TRIALS}

Advances in the treatment of multiple sclerosis depend on clinical trials because of the highly variable and unpredictable course of the disease and the difficulty in precisely measuring neurologic disability. Because progression of the disease in general is slow, these clinical trials traditionally require a relatively large number of patients and long follow-up.

The past decade has seen an increasing ability to perform preliminary examination of the effect of new treatments by using MRI as an outcome measure. The advantage of MRI is its high reproducibility and its high sensitivity in detecting disease activity, which is 5- to 10fold more frequent than clinical relapse. ${ }^{5}$

\section{TREATMENT}

The possibilities for the treatment of multiple sclerosis depend on the clinical situation. We address separately treatment of relapse and disease-modifying treatment in patients with relapsing-remitting disease and those with the secondary progressive phase of the disease.

\section{Treatment of relapse}

The treatment of relapse is irrespective of whether it occurs in patients with relapsing-remitting disease or those with the secondary progressive phase of the disease. Although almost all patients with relapse show some degree of spontaneous recovery, most clinicians advise treatment of relapse that has an important effect on function. For many years, corticosteroids have been the first-choice treatment. Corticosteroids shorten the duration of the relapse and accelerate recovery, but there is no convincing evidence that the overall degree of recovery or the long-term course of the disease is affected.

The most commonly applied regimen consists of a brief course of high-dose methylprednisolone sodium succinate given intravenously IV (500-1,000 mg per day for 3-5 days). Some clinicians substitute oral prednisone for IV methylprednisolone because it is easier to use and costs less. Data substantiating the comparable benefits of oral prednisone and IV methyprednisolone in acute relapses have been presented but are not definitive. ${ }^{6}$ In various studies—all of them small—different dosage regimens of oral steroids have been applied. ${ }^{7}$

\section{Disease-modifying treatment of relapsing-remitting multiple sclerosis}

The goal of treatment in patients with relapsing-remitting multiple sclerosis is to reduce the frequency and severity of relapses (and thereby prevent exacerbations) and to prevent or postpone the onset of the progressive phase of the disease. To achieve this goal, in the past especially, immunosuppressive drugs were used, but they have never found widespread acceptance owing to limited efficacy and considerable toxicity.

More recently, large randomized controlled trials have been performed successfully with interferon beta- $1 \mathrm{a}$, interferon beta- $1 \mathrm{~b}$, and glatiramer acetate. ${ }^{8-11}$ These substances should be seen as immune modulators rather than immune suppressors. The trials have led to the regulatory approval of 4 agents-Avonex, Biogen, United States; Betaseron, Berlex, United States (Betaferon, Schering, Germany); Copaxone, Teva, Israel; and Rebif, Serono, Switzerland-for reducing the severity and frequency of relapses (table).

\section{Interferon beta}

Currently 2 forms of recombinant interferon beta, interferon beta- $1 \mathrm{a}$ and interferon beta- $1 \mathrm{~b}$, have been approved by US and European regulatory authorities for the treatment of relapsing-remitting multiple sclerosis. Interferon beta-1a (Avonex, Rebif) is a glycosylated, recombinant product from mammalian cells, with an amino acid se- 


\begin{tabular}{|c|c|c|c|c|c|}
\hline Trial & Agent & Drug name & Disease type & Primary outcome & $\begin{array}{l}\text { Result on } \\
\text { primary outcome }\end{array}$ \\
\hline Jacobs et al, $1998^{8,16}$ & $\begin{array}{l}\text { Interferon beta-1a } \\
\text { Interferon beta-1a } \\
\text { Interferon beta-1a } \\
\text { Interferon beta-1a }\end{array}$ & $\begin{array}{l}\text { Avonex } \\
\text { Avonex } \\
\text { Avonex } \\
\text { Avonex }\end{array}$ & $\begin{array}{l}\text { Relapsing-remitting } \\
\text { Secondary progressive } \\
\text { After 1st attack } \\
\text { Primary progressive }\end{array}$ & $\begin{array}{l}\text { Time to confirmed progression } \\
\text { Time to confirmed progression } \\
\text { Time to } 2 \text { nd attack } \\
\text { Time to confirmed progression }\end{array}$ & $\begin{array}{l}\text { Positive } \\
\text { Trial still ongoing } \\
\text { Positive } \\
\text { Presented as negative; } \\
\quad \text { not yet published }\end{array}$ \\
\hline PRISMS Study Group, $1998^{9}$ & Interferon beta-1a & Rebif & Relapsing-remitting & Relapse rate & Positive \\
\hline SPECTRIMS study* & Interferon beta-1a & Rebif & Secondary progressive & Time to confirmed progression & Presented as negative \\
\hline Comi et al, $1995^{15}$ & Interferon beta-1a & Rebif & After 1st attack & Time to 2 nd attack & $\begin{array}{l}\text { Presented as positive; } \\
\text { not yet published }\end{array}$ \\
\hline $\begin{array}{l}\text { INFB Multiple Sclerosis } \\
\text { Study Group, } 1993\end{array}$ & Interferon beta-1b & Betaseron & Relapsing-remitting & Relapse rate & Positive \\
\hline European Study Group, $1998^{20}$ & $\begin{array}{l}\text { Interferon beta-1b } \\
\text { Interferon beta-1b }\end{array}$ & $\begin{array}{l}\text { Betaseron } \\
\text { Betaseron }\end{array}$ & $\begin{array}{l}\text { Secondary progressive } \\
\text { Secondary progressive }\end{array}$ & $\begin{array}{l}\text { Time to confirmed progression } \\
\text { Time to confirmed progression }\end{array}$ & $\begin{array}{l}\text { Positive } \\
\text { Presented as negative; } \\
\text { not yet published }\end{array}$ \\
\hline Johnson et al, $1995^{11}$ & Glatiramer acetate & Copaxone & Relapsing-remitting & Relapse rate & Positive \\
\hline
\end{tabular}

*Abstract only; not yet published.

quence identical to that of natural interferon beta. Interferon beta- $1 \mathrm{~b}$ (Betaseron [Betaferon]) is a nonglycosylated recombinant product from bacterial cells in which serine is substituted for cysteine at position 17.

All 3 drugs have been studied in large double-blind placebo-controlled randomized clinical trials. ${ }^{8-10}$ Inclusion in these studies was restricted to patients with clinically active disease in the years before entry to the study (2 exacerbations in 2 years for Betaseron and Rebif; 2 in 2 years for Avonex) who had mild to moderate disability and essentially were fully ambulatory (expanded disability status scale, 0-5.5 for Betaseron; 0-5 for Rebif; and 1-3.5 for Avonex). The most prominent clinical result of all trials was a clear reduction in both frequency (by about a third) and severity of exacerbations. These observations with regard to treatment effect were supported by convincing findings on MRI both as a reduction of active lesions and as a positive effect on total lesion load in the brain. ${ }^{12-14}$ Because of the robustness of the evidence, most experts consider interferon beta as first-choice treatment in

\section{Unresolved issues in treatment with interferon beta}

- Optimal moment of initiation of treatment

- Optimal dose, frequency, and route of administration

- Long-term effects of treatment

- When to stop treatment

- Occurrence and relevance of neutralizing antibodies

- Mechanism of action

- Cost utility patients with relapsing-remitting multiple sclerosis. Many issues related to treatment with interferon beta, however, remain unresolved (box).

\section{When to start and stop interferon beta}

In individual patients, decisions on the initiation of treatment should be based on the course of the disease, but about $10 \%$ to $20 \%$ of patients have relatively benign disease, so they may not require disease-modifying treatment. Treatment should not be postponed until after persistent neurologic deficits have occurred because interferon beta does not reverse fixed deficits. Disease-modifying treatment should be considered early in the course of disease for patients with an unfavorable prognosis, but the rate and pattern of progression of disease cannot be reliably predicted at initial assessment. Whether long-term treatment should start at the time of the first attack, which seems to be sensible for preventive therapy, was addressed in 2 placebo-controlled studies. ${ }^{15,16}$ These studies showed that low-dose interferon beta-1a reduces the likelihood of a second episode in the 2 or 3 years following a first episode suggestive of multiple sclerosis in patients who have prognostically unfavorable findings on MRI.

Most guidelines concerning treatment with interferon beta in relapsing-remitting multiple sclerosis are based on the inclusion criteria that have been used in the placebocontrolled trials mentioned above. Patients with definite relapsing-remitting disease who have had at least 2 relapses in the past 2 or 3 years and who are still able to walk without support for at least $100 \mathrm{~m}$ are considered eligible for treatment. It is extremely important that before these long-term treatments are implemented, counseling about 
realistic objectives, both regarding efficacy and side effects, takes place because overly optimistic expectations may complicate treatment.

Whether treatment should be discontinued at some time is currently unknown because limited information is available on the long-term effects of interferon beta. Current guidelines on stopping treatment are related to side effects, desire to become pregnant, and perceived inefficacy as shown by frequent relapses or progression of disability during treatment.

\section{Choice of drug}

Direct comparisons have not been made between the different interferon beta preparations, and therefore, definite conclusions from the published data about the superiority of 1 preparation over the other are not possible. The main differences between the registered drugs are the amount of interferon beta given and the route and frequency of administration: Avonex, 6 million units $(30 \mu \mathrm{g})$ by intramuscular injection once a week; Betaseron, 8 million units $(250 \mu \mathrm{g})$ by subcutaneous administration every other day; and Rebif, 6 million units $(22 \mu \mathrm{g})$ by subcutaneous administration 3 times a week. Treatment with any of these drugs is usually well tolerated.

One study compared 3 dosages of interferon beta-1a given subcutaneously once a week with placebo and showed increasing treatment effect with increasing dosage, thereby suggesting that some of the currently applied dose regimens might be suboptimal. ${ }^{17}$

\section{Cost utility}

Quality of life has been shown to be substantially reduced in patients with multiple sclerosis, and it would be important to know whether interferon beta has a favorable effect on quality of life. Although it is likely that a reduction in frequency and severity of attacks makes a difference to the quality of life of a patient with multiple sclerosis, studies have so far not provided firm evidence for this. Models based on assumptions (on costs and savings) and estimates of long-term gains have been described, concluding that treatment with interferon beta has a high cost per qualityadjusted life-years gained. ${ }^{18}$

\section{Glatiramer acetate}

Glatiramer acetate (Copaxone) is a synthetic copolymer with some immunologic similarities to myelin basic protein, 1 of the major components of myelin. Daily treatment with subcutaneous administration of $20 \mathrm{mg}$ of glatiramer acetate resulted in a $29 \%$ reduction of the annual relapse rate in a 2-year trial. ${ }^{11}$ These clinical observations were later supported by findings on MRI in a separate study. ${ }^{19}$ Adverse effects of glatiramer acetate are usually mild. Definitive data on the effect of glatiramer acetate on disease progression are not available.

Glatiramer acetate was approved by the US authorities in 1996, but so far there is no pan-European license. The indications for the use of glatiramer acetate are comparable to those for interferon beta, but most clinicians consider it as second-line treatment of relapsing-remitting multiple sclerosis.

\section{Disease-modifying treatment of secondary progressive multiple sclerosis}

The goal of treatment in patients with secondary progressive multiple sclerosis is to prevent progressive worsening of the disease. Until recently, no agent had a favorable effect on the disease once it had entered the secondary progressive phase.

\section{Interferon beta}

A randomized, double-blind, placebo-controlled, multicenter trial of interferon beta-1b (8 million units subcutaneously every other day) was recently completed in Europe, including 718 patients with clinically active secondary progressive multiple sclerosis. ${ }^{20}$ At the predetermined interim analysis, the study was stopped because of a significant difference in the time to confirmed neurologic deterioration in favor of the treated group. The delay of progression was about 9 to 12 months. Significant reductions were also observed in time to become wheelchair bound, number of steroid courses given, and number of admissions to a hospital because of multiple sclerosis. Based on these results, interferon beta- $1 \mathrm{~b}$ was approved for use in patients with secondary progressive multiple sclerosis in Europe.

Recently it was reported that in a large placebocontrolled trial, interferon beta-1a (Rebif) failed to have a major effect on disease progression (the Secondary Progressive Efficacy Clinical Trial of Recombinant Interferon Beta-1a in Multiple Sclerosis [SPECTRIMS] study, 9th meeting of the European Neurological Society, Milan, 1999). So far, these results have been published only in abstract form. Full publication of the results is eagerly awaited, as are the detailed results of a second study of interferon beta-1b in patients with secondary progressive multiple sclerosis that was carried out in the United States.

The question of when to start and stop interferon beta in secondary progressive disease is difficult to answer; evidence should be reviewed as the detailed results of further trials become available (table). It is likely that these results will also have an effect on the outcome of cost-utility studies in secondary progressive multiple sclerosis. ${ }^{21}$

\section{Role of traditional immunosuppressants}

Given that both interferon beta and glatiramer acetate, although convincingly shown to be effective, have major limitations, including cost, inconvenience (given parenterally), and a relatively modest overall effect on disease course, several experts have urged reconsideration of the role of immunosuppressants like azathioprine or metho- 
trexate. Compared with interferon and glatiramer acetate, these drugs are much cheaper, easier to give, and might also have a favorable effect on the natural course of the disease. ${ }^{22,23}$ The lack of convincing data for immunosuppressants from MRI — as opposed to interferon beta and glatiramer acetate- has probably contributed to their rather modest acceptance. This is especially important now that MRI has achieved more widespread acceptance as a surrogate marker of disease progression owing to better understanding of its correlation with both clinical disability and underlying pathology of disease.

\section{NEW DEVELOPMENTS}

A whole range of new treatment strategies is under investigation, varying from subtle immune interventions, such as the induction of immune tolerance or administration of various monoclonal antibodies, to aggressive strategies such as bone marrow transplantation. ${ }^{24}$ Recent observations of axonal damage early in the disease course and increasing disability despite optimal anti-inflammatory treatment emphasize the need for rigorous investigation of neuroprotective treatment. ${ }^{25-27}$

Many experts think that various treatment strategies should be combined to be optimally effective. Alternatively, if preliminary neuropathologic observations indicating that individual patients may have unique mechanisms underlying their disease process would be confirmed, it could be possible in the future to tailor treatment on the basis of individual patient characteristics. ${ }^{28}$

\section{CONCLUSIONS}

The introduction of interferon beta and glatiramer acetate as drugs effective in modifying the course of relapsingremitting multiple sclerosis has improved the treatment of multiple sclerosis and provided tools to unravel further the mechanisms of the disease.

References

1 Weinshenker BG. The natural history of multiple sclerosis. Neurol Clin 1995;13:119-146.

2 Oksenberg JR, Hauser SL. New insights into the immunogenetics of multiple sclerosis. Curr Opin Neurol 1997;10:181-185.

3 Lucchinetti CF, Bruck W, Rodriguez M, Lassmann H. Distinct patterns of multiple sclerosis pathology indicates heterogeneity on pathogenesis. Brain Pathol 1996;6:259-274.

4 Miller DH, Albert PS, Barkhof F, et al. Guidelines for the use of magnetic resonance techniques in monitoring the treatment of multiple sclerosis: US National MS Society Task Force. Ann Neurol 1996;39:6-16

5 Miller DH, Grossman RI, Reingold SC, McFarland HF. The role of magnetic resonance techniques in understanding and managing multiple sclerosis. Brain 1998;121(pt 1):3-24.

6 Barnes D, Hughes RA, Morris RW, et al. Randomised trial of oral and intravenous methylprednisolone in acute relapses of multiple sclerosis. Lancet 1997;349:902-906.

7 Uitdehaag BMJ, Barkhof F. Methylprednisolone for acute relapses of multiple sclerosis: can oral replace intravenous administration? CNS Drugs 1998;10:233-238.

8 Jacobs LD, Cookfair DL, Rudick RA, et al. Intramuscular interferon beta-1a for disease progression in relapsing multiple sclerosis. Ann Neurol 1996;39:285-294.
9 PRISMS (Prevention of Relapses and Disability by Interferon beta-1a Subcutaneously in Multiple Sclerosis) Study Group. Randomised double-blind placebo-controlled study of interferon beta-1a in relapsing/remitting multiple sclerosis. Lancet 1998;352:1498-1504 [published erratum appears in Lancet 1999;353:678].

10 The IFNB Multiple Sclerosis Study Group. Interferon beta-1b is effective in relapsing-remitting multiple sclerosis: I, clinical results of a multicenter, randomized, double-blind, placebo-controlled trial. Neurology 1993;43:655-661.

11 Johnson KP, Brooks BR, Cohen JA, et al. Copolymer 1 reduces relapse rate and improves disability in relapsing-remitting multiple sclerosis: results of a phase III multicenter, double-blind placebo-controlled trial: the Copolymer 1 Multiple Sclerosis Study Group. Neurology 1995; $45: 1268-1276$

12 Paty DW, Li DKB, for the UBC MS/MRI Study Group and the IFNB Multiple Sclerosis Study Group. Interferon beta-1b is effective in relapsing-remitting multiple sclerosis: II, MRI analysis results of a multicenter, randomized, double-blind, placebo-controlled trial. Neurology 1993;43:662-667.

13 Li DKB, Paty DW, for the Prevention of Relapses and Disability by Interferon beta-1a Subcutaneously in Multiple Sclerosis. Magnetic resonance imaging results of the PRISMS trial: a randomized, double-blind, placebo-controlled study of interferon- $\beta 1 \mathrm{a}$ in relapsing-remitting multiple sclerosis. Ann Neurol 1999;46:197-206.

14 Simon JH, Jacobs LD, Campion M, et al, for the Multiple Sclerosis Collaborative Research Group. Magnetic resonance studies of intramuscular interferon beta-1a for relapsing remitting multiple sclerosis. Ann Neurol 1998;43:79-87.

15 Comi G, Barkhof F, Durelli L, et al. Early treatment of multiple sclerosis with Rebif (recombinant human interferon beta): design of the study. Mult Scler 1995;1:S24-S27.

16 Jacobs LD, Beck RW, Simon JH et al. Intramuscular interferon beta-1 therapy initiated during a first demyelinating event in multiple sclerosis. N Engl J Med 2000;343:898-904.

17 The Once Weekly Interferon for MS Study Group. Evidence of interferon beta-1a dose response in relapsing-remitting MS: the OWIMS study. Neurology 1999;53:679-686.

18 Parkin D, Jacoby A, McNamee P, Miller P, Thomas S, Bates D. Treatment of multiple sclerosis with interferon $\beta$ : an appraisal of cost-effectiveness and quality of life. J Neurol Neurosurg Psychiatry 2000;68:144-149.

19 Comi G, Filippi M, for the Copaxone Magnetic Resonance Imaging Study Group. The effect of glatiramer acetate (Copaxone) on disease activity as measured by cerebral MRI in patients with relapsing-remitting multiple sclerosis (RRMS): a multicenter, randomized, double-blind, placebo-controlled study extended by open label treatment [abstract]. Neurology 1999;52(suppl 2):A289.

20 European Study Group on interferon beta-1b in secondary-progressive MS. Placebo-controlled multicentre randomised trial of interferon beta-1b in treatment of secondary progressive multiple sclerosis. Lancet 1998;352:1491-1497.

21 Forbes RB, Lees A, Waugh N, Swingler RJ. Population based cost utility study of interferon beta- $1 \mathrm{~b}$ in secondary progressive multiple sclerosis. BMJ 1999;319:1529-1533.

22 Yudkin PL, Ellison GW, Ghezzi A, et al. Overview of azathioprine treatment in multiple sclerosis. Lancet 1991;338:1051-1055.

23 Goodkin DE, Rudick RA, VanderBrug Medendorp S, et al. Low-dose $(7.5 \mathrm{mg})$ oral methotrexate reduces the rate of progression in chronic progressive multiple sclerosis. Ann Neurol 1995;37:30-40.

24 Hohlfeld R. Biotechnological agents for the immunotherapy of multiple sclerosis: principles, problems and perspectives. Brain 1997;120(pt 5):865-916.

25 Trapp BD, Peterson J, Ransohoff RM, Rudick R, Mork S, Bo L. Axonal transection in the lesions of multiple sclerosis. $N$ Engl J Med 1998;338:278-285.

26 Paolillo A, Coles AJ, Molyneux PD, et al. Quantitative MRI in patients with secondary progressive MS treated with monoclonal antibody Campath 1H. Neurology 1999;53:751-757.

27 Coles AJ, Wing MG, Molyneux P, et al. Monoclonal antibody treatment exposes three mechanisms underlying the clinical course of multiple sclerosis. Ann Neurol 1999;46:296-304.

28 Lucchinetti C, Bruck W, Parisi J, Scheithauer B, Rodriguez M, Lassmann H. A quantitative analysis of oligodendrocytes in multiple sclerosis lesions: a study of 113 cases. Brain 1999;122(pt 12):2279-2295. 